\title{
Ekzemkinder brauchen Sonne und Meer
}

\author{
Kindern mit atopischem Ekzem hilft häufig ein Klimawechsel, etwa \\ ein Aufenthalt am Meer. Auch ein Wechsel in wärmere Gefilde hat \\ positive Auswirkungen. Diese langjährigen Erfahrungen wurden nun \\ in einer randomisierten kontrollierten Studie überprüft.
}

n Norwegen zeigen epidemiologische Studien eine hohe Assoziation zwischen atopischem Ekzem und Außentemperatur bzw. Besonnungszeit: Im Norden des Landes liegt die Lebenszeitprävalenz für das atopische Ekzem bei 23,6\%, im Süden bei 12,7\%. Seit 1998 ermöglicht das norwegische Gesundheitsministerium so genannte „Gesundheitsreisen“, bei denen Kinder mit Neurodermitis für etwa einen Monat in eine subtropische Klimazone, beispielsweise auf die Kanaren, geschickt werden.

Um den Erfolg dieser Maßnahme zu überprüfen, wurden randomisiert 30 Kinder mit schwerem atopischen Ekzem für vier Wochen auf die Kanarischen Inseln verschickt, 26 andere Kinder verblieben zu Hause in Norwegen in ihrer gewohnten Umgebung und dienten als Kontrollgruppe. Beide Gruppen wurden über drei Monate nachbeobachtet, primäres Zielkriterium waren Veränderungen im SCORAD-Index.
Bei den Kindern mit Aufenthalt auf den Kanaren fiel der SCORAD von 37,2 zu Beginn auf 12,2 am Ende der Kur und lag nach drei Monaten bei 21,2 $(\mathrm{p}<0,0005)$, er reduzierte sich damit deutlich mehr als bei den Kontrollen. Der Children's Dermatology Life Quality Index, ein sekundärer Studienpara-

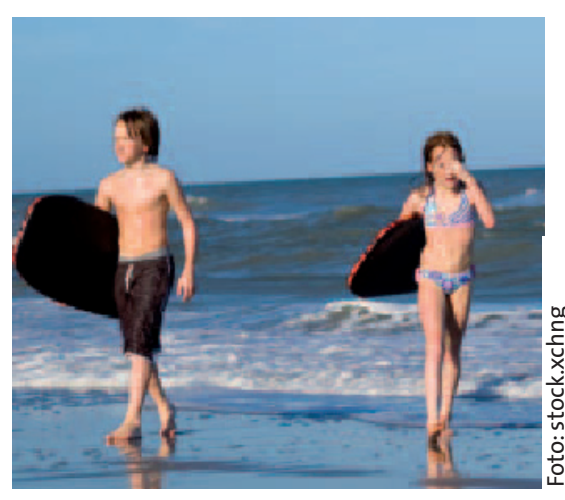

Die Sonnenexposititon sowie das Baden im Meer beeinflussen offensichtlich das atopische Ekzem positiv.

\section{Calcineurininhibitor im Langzeittest}

\section{Der Calcineurininhibitor Tacrolimus ist bei der Behandlung akuter Phasen einer atopischen Dermatitis eine Alternative zu topischen Glukokortikoiden. Nachdem sich die Substanz bei Erwachsenen auch über einen längeren Zeitraum als sicher erwiesen hat, wurde die Lang- zeitverträglichkeit jetzt bei Kindern überprüft.}

E in internationales Studienteam dokumentierte für eine offene Studie die Daten von 466 Kindern zwischen zwei und 15 Jahren mit einer atopischen Dermatitis. Die jungen Probanden trugen während der akuten Phasen der Erkrankung 0,03\%ige oder 0,1\%ige Tacrolimus-Salbe zweimal täglich auf die betroffenen Hautstellen auf. Non-Responder wurden ausgeschlossen. Die Beobachtungsdauer betrug bis zu 29,5 Monate, primärer Endpunkt war die Inzidenz unerwünschter Arzneimittelwirkungen.

Die Beobachtung dauerte im Durchschnitt 16,3 Monate, wobei die Tacrolimus-Salbe an durchschnittlich 63,7\% der Studientage angewendet wurde. 15 Patienten (3,2\%) brachen die Studie aufgrund von Nebenwirkungen ab. Als häufigste Nebenwirkungen wurden Brennen und Juckreiz an den Applikationsstellen genannt, die Häufigkeit nahm im Laufe meter, verbesserte sich in der Interventionsgruppe von 8,7 auf 2,2 nach vier Wochen und auf 4,5 nach drei Monaten $(\mathrm{p}<0,0005)$, in der Kontrollgruppe ergaben sich keine Veränderungen. Auch der Anteil der Kinder mit einer Kolonisation der Haut mit Staphylococcus aureus ging in der Kurgruppe von 23/30 $(77 \%)$ auf $12 / 30(40 \%)$ nach einem Monat zurück $(\mathrm{p}=0,001)$ und verblieb so auch nach drei Monaten $(\mathrm{p}=0,005)$. Außerdem konnte der Gebrauch von topischen Glukokortikoiden in der Kurgruppe reduziert werden, nicht dagegen in der Kontrollgruppe.

Fazit: Kinder mit schwerem atopischen Ekzem erleben nach einem Klimawechsel von Norwegen auf die subtropischen Kanaren eine Verbesserung der Hautsymptomatik und der Lebensqualität. Der Effekt geht nur langsam zurück, so dass auch nach drei Monaten noch Verbesserungen gegenüber dem Ausgangswert bestehen. Die Risiken der Sonnenexposition im Kindesalter für die Entwicklung maligner Hauttumoren sollten bei der Bewertung dieser Ekzemtherapie Berücksichigung finden

Byremo G et al. Effect of climatic change in children with atopic eczema. Allergy 2006; 61: 1403-10

der Zeit ab. Die Inzidenz viraler Infekte im Beobachtungszeitraum entsprach der bei hautgesunden Kindern. Hinweise auf eine Wachstumsretardierung oder Hautatrophie ergaben sich nicht. Es wurde nach zweiwöchiger Anwendung eine deutliche Verbesserung des Hautzustandes gesehen, die über die gesamte Studienzeit anhielt.

Fazit: Die Langzeitanwendung von Tacrolimus-Salbe bei Kindern ab zwei Jahren mit atopischer Dermatitis ist gut verträglich. Vor allem zu Beginn der Therapie können Brennen und Jucken der behandelten Stellen auftreten.

$b k$

Remitz A et al. Long-term safety and efficacy of tacrolimus ointment for the treatment of atopic dermatitis in children. Acta Derm Venereol 2007; 87: 54-61 